\title{
Reflexión, acción, decisión: trayectorias en la construcción de la identidad de género en el patriarcado gitano
}

\author{
Reflexion, action and decision: trajectories in the construction of \\ gender identity in gipsy patriarchy
}

\author{
María Esther López Rodríguez \\ Universidad de Zaragoza \\ estherlo@unizar.es(ESPAÑA)
}

Alexia Sanz Hernández

Universidad de Zaragoza

Recibido: 05.022017

Aceptado: 24.07.2017

\section{RESUMEN}

Este artículo recoge una versión del momento actual de tensión, cambio y emergencia de individualidad femenina entre mujeres gitanas zaragozanas. Se partió de la premisa de que la convergencia de atributos personales como la identidad y el género (a la vez conceptos clave de la construcción individual), suponían un acicate a la estructura patriarcal -identidad étnica-, entendiendo que cuando la mujer toma conciencia de sí -identidad de género-, emerge la tensión entre el paradigma tradicional y el moderno. La aproximación cualitativa y biográfica (sustentada en la narración y conversación, la observación participante y el análisis cualitativo a partir de la teoría fundamentada) nos ha permitido analizar desde dos dimensiones claves, la psicológica y la contextual, el recorrido introspectivo secuencial por el que avanzan (reflexión-decisión-acción) y las trayectorias vitales que se van conformando al intentar encajar el crecimiento personal con la no afectación de la estructura patriarcal gitana, su cultura y su sistema de valores y mandatos. 


\title{
PALABRAS CLAVE
} ción.

Identidad, género, trayectoria, narración biográfica, reflexión/decisión/ac-

\begin{abstract}
This article collects a version of the current moment of tension, change and emergence of feminine individuality among gypsy women from Zaragoza. It was based on the premise that the convergence of personal attributes such as identity and gender (both key concepts of the individual construction) were a challenge to the patriarchal structure -ethnic identity- understanding that when women become aware of herself -gender identity- the tension between the traditional and the modern paradigm emerges.

The qualitative and biographical approach (based on narrative and conversation, participant observation and qualitative analysis based on grounded theory) has allowed us to analyze from two key dimensions, the psychological and the contextual, the sequential introspective path through which they advance (reflection-decision-action) and the vital trajectories that are conforming when trying to fit personal growth with the non-affectation of the patriarchal gypsy structure, its culture and its system of values and mandates.
\end{abstract}

\section{KEY WORDS} tion.

Identity, gender, trajectory, biographical narration, reflection/ decision/ac-

\section{INTRODUCCIÓN}

La presente investigación es fruto de una coincidencia en el espacio-tiempo, y de una observación continuada con vínculos de distinto nivel, que ha posibilitado el establecimiento de una relación dialógica con mujeres gitanas en la ciudad de Zaragoza. Dicha relación ha ido explicitando tres ideas fuerza.

La primera se relaciona con el hecho de que diariamente las mujeres gitanas, en su ir y venir entre el adentro y el afuera de su cotidianidad -gitanidad-, cargan y arrastran una mochila de identidad que determina su peso en la intra estructura relacional étnica.

La segunda se refiere a que ese recorrido determina una línea de cruce que hace emerger una tensión representada en el transitar entre paradigmas convergentes: el tradicional gitano y el moderno de la sociedad mayoritaria.

Y la tercera destaca que la emergencia de la individualidad -de la mujer gitana y de los jóvenes- es un foco de tensión sistémica que exige reflexividad 
durante el proceso de toma de decisiones en torno a lo que hemos denominado: cruzar la línea ${ }^{1}$.

Las tres ideas fuerza convergen en una principal que es la construcción de la individualidad y de la identidad de género durante ese proceso de cruzar la línea, mediado por una necesaria mirada reflexiva descifradora de los significados emergentes en los momentos de tensión inherentes a la ineludible toma de decisiones.

A partir de la asunción de una perspectiva epistemológica y metodológica de corte cualitativo (Denzin y Lincoln, 2011), se realizaron entrevistas semiestructuradas -que generaban narrativas biográficas o relatos de vida (Sanz, 2005; Vallés, 1997; Pujadas, 2002; Ibáñez, 2007; García-Huidobro, 2016)- a 18 mujeres gitanas; si bien para el análisis del material recogido se recurrió a herramientas de segunda generación de la Grounded Theory (Charmaz, 2010). Todo ello nos ha permitido descifrar las trayectorias de cambios personales que impactan en la estructura relacional gitana y confrontan las tensiones individuales ligadas al despertar de una conciencia emic femenina en una dialéctica entre el ser y deber ser que la construcción de la identidad de género plantea al sujeto emergente. En ese contexto, sin pretender subvertir el sistema de valores ni despegarse de la identidad étnica, la mujer gitana aboga por una revisión y re-significación de esas dinámicas y un encaje de la individualidad a nivel intragrupal, vivenciando, a la par, una crisis interior de carácter conflictual.

Desde esa dimensión personal, el conflicto se ubica durante el trayecto desde el adentro al afuera, mientras que la tensión se aloja en la dialéctica personalcontextual en el trayecto de regreso (cruzando la línea), caracterizado por la percepción en el intragrupo del riesgo de desidentificación o apayamiento ${ }^{2}$ y a la par la demanda al exogrupo -en el paradigma moderno en la sociedad mayoritaria- de un reconocimiento real, inclusivo y participativo de la identidad cultural gitana, como solución definitiva para legitimar el cambio individual y resolver la tensión intra grupal.

De la resolución de esas tensiones va el mencionado proceso de construcción de la identidad personal en clave de género en un tempo propio visibilizado en y visibilizador de la emergencia de la individualidad.

En este artículo vamos a detallar las perspectivas de análisis que han guiado la investigación y el modelo metodológico, a la par que se repasan las diferentes fuentes que han contribuido a conformar el marco teórico y analítico, y se hacen algunos apuntes necesarios sobre la identidad cultural gitana y el sistema de valores estructurante. Finalmente, presentaremos lo que hemos denominado "inconclusiones", ahondando en la complejidad de las trayectorias identifica-

${ }^{1}$ La línea es un espacio de intercambio simbólico y cotidiano: el umbral de la puerta de una casa que conduce al afuera y devuelve al adentro en el transitar diario de una mujer gitana (y de todas). En una dinámica de avances y retrocesos, este espacio tan poco trascendente representa la socialización en todas sus fases: de casa a los propios; de casa a los otros; de casa al yo. Además el orden de identidades no es casual porque lo cotidiano es su terreno.

${ }^{2}$ N.de i. Apayamiento estigmatización/acusación que refieren sufrir los miembros de la comunidad que adquieren hábitos extra grupales (lenguaje, costumbres, vínculos...). 
das, la incertidumbre durante la toma de decisiones y la gestión del tiempo para afrontarla.

\section{PERSPECTIVAS DE ANÁLISIS}

Abordar un complejo identitario como el de la identidad cultural gitana, requiere una profundización en los distintos aspectos donde se asienta su historia, un repaso de los fundamentos de su sistema de valores cohesivo y una revisión de los pilares que construyen su andamiaje estructural relacional. Ello explica que para esta investigación recurriéramos a tres perspectivas complementarias, la histórico-jurídica, la antropológica y la sociológica, siendo esta última el eje sobre el que gira la interpretación de resultados.

Desde la perspectiva histórico-jurídica, hemos dado por buena la teoría de que los gitanos y gitanas, como toda minoría étnica ${ }^{3}$, tienen conciencia de diferencia y de identidad cultural (San Román, 1994; Rothea, 2008; Martínez, 2007), asentada en la creencia de un origen común ubicado en la India (Aguirre, 2006), y comparten un sistema de valores culturales, impregnados de cultura mayoritaria, pero con rasgos muy característicos que intentan conservar (San Román, 1976, 1997; Rodríguez, 2008). Esta conciencia de grupo distinto, les hace reivindicar su diferencia (Ramírez, 1971; Leblon, 1985; Fonseca, 2009) y por tanto reafirmar una identidad cultural transterritorial o panétnica (Prieto, 2007; Flecha, Mircea y Sordé, 2013: 1-16).

La relación gitanos-no gitanos, o minoría-mayoría, ha estado marcada por dos épocas muy diferenciadas que han determinado la dinámica relacional a ambos lados de la línea: Una época "oscura" con mecanismos institucionalizados de persecución e intentos de asimilación que parte desde la primera pragmática de los Reyes Católicos en 1499 y se acerca hasta la instauración de la democracia con la derogación de la Ley de Vagos y Maleantes tras la Transición española (Aguirre, 2006; Jiménez, 2008, 2011; Heredia, 2012). Y otra "luminosa" con mecanismos de inclusión y diseño de políticas identitarias de reconocimiento creciente (FSG, 2011; FSG y Laparra, 2006; FSG y EDIS, 2009).

Desde la perspectiva antropológica se analizaron el constructo cultural focalizando sobre el rol y la posición de las mujeres en la estructura, para interpretar los significados de lo que hemos denominado "mandatos"4 sostenedores del sistema de valores agrupado bajo las expresiones familia y solidaridad orgánica.

${ }^{3}$ La comunidad gitana es la minoría étnica más grande de Europa, reconocida a nivel cultural, institucional y social. Más de doce millones de gitanos viven desperdigados por todo el continente. Un sentimiento de identidad referenciada en la cultura refuerza la cohesión del grupo en un sentido de pertenencia étnica que exalta la gitanidad, o la romipen o perspectiva de la vida.

${ }^{4}$ Los hemos sistematizado en dos grupos: 1) Mandatos de género y rol del Valor Familia: sumisión al varón; obediencia; respeto; cumplimiento de ritos de paso: pedimiento, boda, maternidad. 2) Mandatos de género y rol del Valor de la Solidaridad Orgánica: cuidado de los propios; soportar la economía familiar; capitalizar beneficios individuales en pos del grupo; y finalmente el sentido instrumental de la convivencia intercultural. 
Así hemos testimoniado que las mujeres gitanas son un valor diferencial en sí mismo dentro de la estructura patriarcal gitana como agente enculturador principal y valedor de la continuidad étnica a través de la reproducción. Lo asumen, lo verbalizan y voluntariamente cumplen con lo que se espera de ellas.

Al mismo tiempo, las mujeres gitanas se autodefinen motores de un cambio (Jiménez, 2010) del que se sienten artífices y protagonistas. Se trata de un cambio en los cimientos de la estructura social de la que emergen y para lo que pretenden compatibilizar la identidad individual y la identidad cultural. Deben sortear las resistencias de los sectores más ortodoxos de la intra sociedad que se erigen como factores de pervivencia identitaria atados a la tradición y que cuesta modificar. Asimismo, deben sortear las demandas de modernización que se imponen desde el exterior, algo que tampoco se puede alterar (Alberdi, 1996; Elboj y Vicén, 2007; Abajo y Carrasco, 2004).

Ser mujer gitana hoy, con toda la carga de transformación que supone adaptarse a los tiempos actuales de tensión sistémica entre valores tradicionales y valores modernos, nos pone frente a sujetos que arrastran la pesada carga que se suma desde ambos lados de la línea (una mochila de identidad). En el interior, la estructura patriarcal impone unos mandatos sujetos a valores cohesivos y determinantes con mecanismos de desigualdad que se asocian a la identidad endogrupal. Desde el exterior se proponen unos mecanismos de relación que tienden a la igualdad (Amorós, 1996; Alberdi, 1996; Bustelo y Lombardo, 2005).

Esta dialéctica bidimensional -contextual y personal- demanda respuestas adaptativas a los estímulos externos e integradoras a las demandas internas; pero de momento las mujeres entrevistadas encuentran incertidumbre durante la reflexividad que atañe a cada decisión. Como en un juego hamletiano, el ser o no ser de la identidad gitana es determinante del ser o no ser del individuo en la intra y extra sociedad. La mujer gitana hoy se debate, en su trayectoria individual, en una carrera desesperada por no perder la identidad de referencia, por no dejar de ser y pertenecer.

Durante las distintas fases de la socialización, la acomodación de estos estímulos minimizará el riesgo de pérdida de sujetos en pos de su individualidad y el encaje de sus relatos será considerado como una versión modernizada de la identidad cultural, la identidad proyecto según Castells (2001). En este punto hemos posicionado el eje tensional principal analizado desde la tercera perspectiva, la sociológica, donde convergen la identidad y el género como atributos de la identidad: la de resistencia que ostenta la minoría gitana frente a la identidad legitimadora de la sociedad mayoritaria. La tensión a ambos lados de la línea se refleja en un escenario donde la identidad proyecto sintetiza ambos atributos en un proceso de construcción de la identidad de género.

La comparación ha permitido hacer una analogía con el momento de cambio que atraviesa la comunidad gitana, durante el encaje de las nuevas individualidades en la socialidad intragrupal. O lo que es lo mismo: aceptar la gitanidad femenina reinterpretada en tono de modernidad. Esto pone al descubierto la tensión infringida al sistema de valores tradicionales dando lugar a una confrontación entre dimensiones de la identidad de resistencia y proyecto: 
- La dimensión contextual de la identidad es la resistencia, que exige al sujeto mujer gitana respeto y sumisión al sistema de valores por razones estructurales y de pervivencia cultural.

- la dimensión personal de la identidad proyecto, es desde donde la mujer gitana reclama aceptación, reconocimiento y un encaje de la nueva concepción femenina en clave de individualidad y género.

Al tiempo se visualiza otro punto de conflicto durante el proceso de intercambio e integración que comporta la relación dialéctica minoría/mayoría, donde la mayoría con su identidad legitimadora estaría validando esta identidad proyecto en fase de adaptación a los valores de la modernidad y a la vez empujaría al endogrupo a resolver su permanencia en la tradición a modo de reconocimiento. Un reconocimiento (Fraser, 2001) que viene de la aceptación de una gitanidad en femenino que impactaría beneficiosamente en la mejora de la calidad de vida del grupo y daría una solución de compromiso al déficit o missrecognition que caracteriza la relación a ambos lados de la línea. Esas serían las voces desde el adentro hacia el afuera que se autodefinen "motor de cambio".

En cuanto al género, las voces que construyen el relato de esta investigación, narran conciencia de género en un discurso sexista solo para ellas, alimentando la tensión que refuerza la tradición patriarcal gitana (sistema de dominación androcéntrica retroalimentada y reforzada en subordinaciones de jerarquías por edad y sexo, dirá Amorós (1985)), defendida por congéneres que perciben, en estas asonadas de individualidad y demandas de reconocimiento, una amenaza al sustrato de la raíz patrilineal o un ataque al valor familia.

Es una encrucijada reflexiva en la construcción identitaria de género que se ha analizado a partir del dilema Wollestoncraft (1792 citada en Lombardo, 2003) entre ser para sí o ser para otros y profundizado en teatralizaciones goffmanianas (1959), artificios de identidad y acciones de resistencia grupal que cobijan o protegen a la mujer gitana ${ }^{5}$. En el intersticio de ese proceso se identificó una dinámica pendular luhmaniana (1997) que oscila entre la autorreflexión y la autopoiesis en los preludios de la decisión y la acción de cruzar la línea ${ }^{6}$.

El relato ansiado en torno a cómo construir la identidad gitana, es el del cruce de líneas que favoreciese el intercambio y la interacción con el exogrupo sin pérdida de referencias y penalizaciones en primer lugar, y en segundo lugar, el de una gestión del tiempo que ya no sería sólo para sí, sino también para los hombres (definidores de la cultura y el sistema de valores), esperando de ellos atisbo de complicidad en la modernización iniciada.

Mirando a las trayectorias que van mudando de paradigmas, conforme cruzan a un lado o al otro de la línea de la cotidianeidad a la socialidad, del espacio

${ }^{5}$ A mayor adhesión a los valores y al cumplimiento de mandatos mayor pureza ascética endogrupal que refuerza la etnicidad y delimita el adentro y el afuera como reforzamiento de la identidad

${ }^{6}$ En los relatos fue importante analizar e interpretar los significados de "los antes" y "los nunca pudieron ser", aspectos imperceptibles para el observador común, pero inexcusables para el investigador. 
privado al público, del sistema de valores tradicional al moderno, se percibe el conflicto que deviene en encrucijada durante el incierto resultado del proceso reflexión-decisión-acción.

\section{EL SUJETO, EL PROBLEMA Y SU CONTEXTO, Y EL MÉTODO.}

\subsection{SOBRE LA MUJER GITANA ZARAGOZANA. PERFILES EN- CONTRADOS Y SELECCIONADOS.}

En Zaragoza se estima que residen unas 12.000 personas gitanas en diferentes barrios de la ciudad, siendo en la Magdalena, el Gancho y Torrero donde están presentes desde hace siglos. Muestran hábitos urbanos análogos a los de clase media baja trabajadora y/o autónoma, de nivel socioeducativo medio y medio-bajo (FSG y Laparra, 2006). En algunos sectores sociales están en el estrato socio-económico bajo, cercano a la pobreza relativa (FOESSA, 2014).

Es una población eminentemente joven -aproximadamente el 50\% son mujeres- inserta en una ciudad moderna (dentro del panorama de ciudades medias españolas), que ha desarrollado algunas políticas sociales y culturales considerando la especificidad de la comunidad gitana. Esto ha contribuido a forjar un movimiento asociativo maduro, relativamente activo $^{7}$, donde el colectivo gitano ha ido ganando cotas de reconocimiento real.

La cercanía con este tejido asociativo permitió tejer una red vincular que facilitó el contacto y selección de las 18 mujeres gitanas participantes en esta investigación, con quienes se pudo conversar y a quienes se ha podido observar a lo largo de 10 años.

Para determinar sus perfiles se tuvieron en cuenta: a) su edad social, desde el concepto de seniority (Mair, 1998), y b) su identificación como gitanas, analizando trayectorias cimentadas por hitos ${ }^{9}$ de estatus y sumisión al poder heteronormador masculino (Amorós, 1985; Pateman, 1987; Platero, 2009).

Se introdujeron las variables tiempo e hito cumplido que conceden la se-

${ }^{7}$ Las más importantes y representativas del colectivo local son la Federación de Asociaciones Gitanas, Fundación Secretariado Gitano y Asociación de Promoción Gitana de Zaragoza.

${ }^{8}$ Las participantes en esta investigación son mujeres residentes en la ciudad de Zaragoza quienes participan activamente de los programas de intervención socio-laboral y dinámicas de búsqueda de empleo en el entorno asociativo gitano. Algunas de ellas, en concreto 7, tienen representatividad étnico-cultural local. La mayoría forman parte de lo que podríamos denominar clase media social y cultural con perfiles que van desde ser empresarias o trabajadoras, estudiantes, amas de casa, divorciadas, solteras, casadas, madres, etc. El contacto continuado ha permitido una observación constante a los sujetos y el fortalecimiento de una interacción sostenida en el tiempo producida en sesiones de orientación laboral, talleres de empoderamiento, dinámicas de grupo, formación continua, alfabetización digital, coordinación de un equipo de azafatas y la relación laboral.

9 Se han considerado el cumplimiento de rituales de paso y el acatamiento de mandatos [matrimonio, maternidad, viudez, etc.] y el respeto a los valores cohesionadores: familia y solidaridad orgánica. 
niority en el grupo; para las más rezagadas en la maduración social y desde el análisis de cohortes (Gil, 1996) en ambos paradigmas, se fijó la mirada en el momento de salto o abismo generacional y la convergencia con las acciones de rebeldía juvenil que hacen identidad. Se analizaron los dilemas que acompañan el proceso de toma de decisiones y que inciden en la modificación de habitus individuales que impactan en lo comunitario (Bourdieu, 1999). En función de la situación personal y el paradigma de referencia serían más o menos significativos las afectaciones al cumplimiento de hitos ${ }^{10}$.

Los perfiles de referente se establecieron en virtud de: a) su posición de respeto intragrupal, b) su liderazgo emergente frente a los seniority para la revisión de mandatos con arreglo a fines orientados a valores.

Las trayectorias sociales se definieron como eje lineal de origen a destino y como trazado con entresijos de idas y venidas, avances, retrocesos, triunfos y derrotas.

\subsection{LAS DIMENSIONES Y VARIABLES DE ANÁLISIS}

Los relatos fueron revelando un foco de tensión entre individualidad y socialidad localizado durante la armonización reconocimiento externo-aceptación interna, tomando esto último como prenda de lealtad al grupo y a sí mismas: ser lo que debo de ser, sin dejar de ser. Con este marco y con el contraste de la observación sostenida en el tiempo por el privilegio de estar allí, cerca (San Martín, 2007) y mediante el modelo de reconstrucción biográfica se fueron encajando los significados en el puzle facetado de la identidad personal a través de la interpretación bidimensional psicológica y contextual (Ibañez, 2007; Sanz, 2005; Pujadas, 2002; Vallés, 1997).

Desde la dimensión psicológica (ser mujer y ser gitana) fueron narrando hitos de promoción y de retrocesos y/o interrupciones, donde refirieron "baches" en las trayectorias o la tensión durante el proceso de toma de decisiones y la gestión del tiempo, valorando la oportunidad y el momento para actuar y avanzar, actuar y retroceder, o actuar y abandonar.

Eran protagonistas de un análisis reflexivo teniendo en cuenta la mirada desde el afuera del grupo (de los propios y los otros ) y desde el adentro individual, considerando como variables intervinientes la resistencia al cambio (eje interpretativo del proceso de construcción individual), en relación con la posición social en la estructural patriarcal gitana, que pone de relieve la dicotómica pendulación tensión/diálogo entre sujeto y grupo, individualidad y socialidad.

Para abordar hermenéuticamente esta dimensión se recurrió a indicadores análiticos como: renuncia, gestión del tiempo, negociación, adaptabilidad selectiva, interrupción, abandono y control social; y para analizar los recorridos de

${ }^{10}$ Se han determinado una serie de hitos fundamentales en las trayectorias modélicas desde el paradigma tradicional donde la boda y la maternidad son clave en el reconocimiento endogrupal y la obtención de la seniority.

EMPIRIA. Revista de Metodología de Ciencias Sociales. N. 38 septiembre-diciembre, 2017, pp. 41-62. ISSN: 1139-5737, DOI/empiria.38.2018.19705 
las diferentes trayectorias como: ocultación de la individualidad, simulación, o teatralización durante la enculturación y el proceso de socialización (Goffman, 1959, 2006).

En segundo lugar, el abordaje desde el nivel contextual (ser mujer gitana), requirió comprender que los cambios individuales han ido alimentando la conflictividad colectiva en dos niveles: grupal (público) y familiar (privado), haciendo responsable al sujeto -en fase de integración de lo contextual externo y armonización con lo interno- de un desajuste interaccional o pérdidas significativas en los espacios de intercambio entre el adentro y el afuera. Fue relevante analizar la tríada reflexión-decisión-acción y las opciones entre determinación individual y control grupal para visualizar el afianzamiento de una emergente conciencia de género. Se remarcaron dos variables para el análisis:

1. El control social legitimador, interpretado a partir de la premisa del atenazamiento de la individualidad por temor a la aculturación y asimilación del sujeto por el exogrupo.

2. El estigma alienante, interpretado a partir del concepto encaje de la individualidad, analizando los distintos grados entre aislamiento o asimilación.

Para la primera variable, se observaron los indicadores: conflictividad familiar, conflictividad intragrupal, conflictividad emocional individual, rechazo del colectivo, o rupturas: Para la segunda, nos centramos en: renuncia al grupo o alejamiento de él, demérito personal, abandono, presión social o desidentificación.

\subsection{EL ANÁLISIS A PARTIR DE LA TEORÍA FUNDAMENTADA. O LA REFLEXIVIDAD INTERPRETATIVA.}

El sujeto de estudio y el contexto exigían creatividad metodológica, reflexividad y revisión de significados captados desde otras miradas hacia el colectivo (Bretones, 2008; Elboj y Vicén, 2007; Abajo y Carrasco, 2005; San Roman, 1987). Esta propuesta intenta ser una aportación a terrenos poco explorados en lo que a la dimensión personal se refiere, recurriendo a la perspectiva inductiva del paradigma de indagación constructivista de la teoría fundamentada (Strauss, 1992; Glaser, 1997; Crabtree y Miller, 1999; Glasser y Corbin, 2002; Trinidad, Carrero y Soriano, 2005; Andreu y Pérez, 2009), para, desde allí, interpretar la versión del momento de cambio y de reflexión identitaria que este relato coral en femenino contenía, identificando categorías analíticas vinculadas al sujeto, a percepciones, a contextos, a información que es más rico obtener mediante esta caja de herramientas analíticas (Andréu, García Nieto y Pérez, 2007). Se han utilizado como fuentes primarias 18 entrevistas biográficas a mujeres gitanas zaragozanas ${ }^{11}$. La codificación y la observación han ido juntas revisando

11 La situación privilegiada de una de las investigadoras dentro del entorno asociativo gitano en constante interacción con las protagonistas del estudio (entre 2007 y 2016) facilitó el contacto 
continuamente los códigos que nutren los conceptos clave: identidad proyecto de género; identidad de resistencia comunitaria; tensión en el cruce de líneas; interseccionalidad.

La conversación y las entrevistas biográficas han permitido oir (y comprender) significados (Vallés, 2014) "atribuidos por los actores" (Schwartz y Jacobs, 2007 citados por Vallés, 2014: 85) para construir un relato del cambio; trayectorias y perspectivas de adaptación de los estímulos a ambos lados de la línea, donde la tensión se situó en la intersección entre dimensiones y paradigmas, en la dialéctica etic-emic durante la tríada reflexión-decisión-acción inherente al proceso de autorreflexión o autopoiesis (Luhman, 1997).

Se partió de una hipótesis principal sobre el cambio social que se está produciendo en la comunidad gitana verbalizado por estas narradoras que abogan por una revisión del sistema de valores y el reequilibrio de fuerzas dentro del sistema sexo-género gitano. Un proceso reflexivo beauvoriano donde estas mujeres se posicionan como imagen y no reflejo en un espejo de identidad enmarcado en el patriarcado. Dos sub-hipótesis fueron guiando la reflexión e interpretación de las señales que se estaban captando:

Por un lado, que la transformación se estaría produciendo en la autodefinición como motor de cambio, lo que estaría configurando un escenario de tensión entre las fuerzas resistentes y mantenedoras del status quo frente a las que desafían a la estructura revisando el vigente sistema de valores como legítima fuente de sentido de la identidad étnica cultural.

Por otro lado, el cambio estaría siendo imperceptible desde el afuera que infligiría una sobreexposición y sobreesfuerzo de los sujetos promocionados que no lograrían capitalizar ni legitimar las narrativas emergentes como argumento de la transformación estructural de la micro sociedad gitana.

La profundidad en la interacción dialógica fue abriendo un abanico de categorías interpretativas del cambio: propuestas de subversión jerárquica y estructural, desafío al control social enculturador, reinterpretaciones del sistema de valores, resignificaciones de los mandatos, entre otras.

La decisión de que el estudio se centrara en la mujer gitana como sujeto inmerso en un contexto tradicional en tensión con la modernidad, las despojó de atributos de liderazgo, promoción exitosa y contexto endogrupal para centrarnos en sus trayectorias identitarias de género en los marcos del patriarcado, viviendo en una ciudad, en un trayecto de ida y vuelta atravesando el umbral de la cotidianeidad: la línea. Tomaron relevancia categorías como el reconocimiento y la

continuado, la observación y la identificación de los perfiles que formaron parte de esta investigación. Para este artículo se han tomado cortas citas literales de varias de las mujeres entrevistadas que relacionamos a continuación, detallando algunos datos personales considerados de relevancia:

M.38: mujer empresaria; referente local; activista religiosa; soltera.

M.29: asalariada; autorreferenciada en fase de ruptura; divorciada sin hijos.

M.30: emprendedora; referente local; activista de género; soltera.

E.27: universitaria; trabajadora; profesional liberal.

R.01: lideresa local; activista étnico-cultural; divorciada; madre.

M.39: emprendedora; alejada del grupo; divorciada; madre. 
aceptación desde las entrañas de la dimensión personal que fueron reconstruyendo y adaptando la resultante de la reflexión. También se captaron los silencios tácticos (Vallés, 2014: 128) ligados a reflexiones profundas identitarias como respuesta velada de oposición al sistema de valores. El sujeto de estudio aportaba información en cada interacción, en un proceso de análisis flexible y dinámico que orientó y enriqueció la metodología cualitativa adoptada, donde la codificación y la observación iban conformando el andamiaje conceptual principal que guiaba el análisis y la reflexión: identidad proyecto de género; identidad de resistencia comunitaria; tensión en el cruce de líneas; interseccionalidad...

En la retroalimentación y la transformación de códigos en los distintos niveles comprensivo e interpretativo, surgió un código central o mayor (Trinidad et al, 2005) para explicar los comportamientos individuales (ante el cruce de línea): "quiero ser yo y seguir con mi cultura". Este podía aglutinar las cuatro opciones que descubrimos al trabajar la tríada reflexión-acción-decisión: 1) el cruce y regreso, 2) cruce y abandono, 3) cruce e interrupción y 4) y último, cruce y ruptura.

La identificación de esta categoría permitió relacionar los códigos con las variables localizadas en la dimensión personal: resistencia al cambio del grupo y control social como sistema penalizador, que incide en la tríada reflexión-accióndecisión determinando los pasos a seguir al cruzar la línea. Se identificaron entonces dos procesos convergentes durante la maduración de la individualidad: uno más vinculado a la fase de construcción identitaria de género, y otro más vinculado a la tensión entre la identidad de resistencia y la identidad proyecto.

Las categorías analíticas que sitúan la posición de las mujeres gitanas dentro de su grupo social (solidaridad orgánica, respeto, sumisión, compromisos de rol y obediencia por un lado, pero también, por otro, y en el contexto de la sociedad mayoritaria, visibilidad, participación, ciudadanía, compromiso de género), se constituyeron en el marco de barreras defensivas (categorías de la identidad de resistencia) construidas por los sujetos dominantes de su etnia frente a los avances en la socialización: trayectorias interrumpidas, matrimonios prematuros, desocialización intergrupal, que han marcado a fuego el contacto intercultural cimentado en una desconfianza mutua. Esta ha construido trincheras de defensa intragrupal ante la "amenaza exterior" y ha dificultado la adaptabilidad a estímulos modernizadores recibidos desde el afuera.

Las dimensiones analíticas de estos relatos evidenciaron una tensión entre los paradigmas moderno y tradicional que anticipábamos como una de las premisas iniciales. El propio proceso de investigación nos acercaba a la aprehensión de la complejidad del recorrido introspectivo secuencial (paralelo a la recuperación histórica), que posicionaba el eje del conflicto en dos niveles: intercultural e intergeneracional, donde el género toma sentido y se refuerza la percepción de minoría frente a la mayoría.

Por otra parte, la reflexión que surge de una mirada a los valores y elementos instituyentes de la identidad cultural gitana vistos desde el adentro tradicional y observados desde el afuera moderno, ha permitido configurar un mapa discursivo identitario que no era sino un relato coral de convergencias y divergencias, que 
nos permitiría reconstruir trayectorias con ejes comunes y discontinuos, que expresamos como pares dicotómicos: avances/retrocesos; promoción/penalización, o autonomía/solidaridad.

Así, desde un nivel comprensivo se fue analizando el discurso resultante de la interacción informal y formal captando señales reveladoras de la posición de género en la estructura patriarcal. A modo de ejemplo podemos describir la consideración del cumplimiento de mandatos como una categoría y una herramienta del sistema de valores, desde donde emergieron códigos del tipo obediencia, aceptación u honramiento; que en un nivel interpretativo se agrupaban en sumisión, retroceso, renuncia, abandono. Realmente, todos los códigos reflejaban una categoría que representaba la tensión entre identidades y al mismo tiempo remitían al contenido de esa mochila imaginaria de la construcción identitaria de género en el patriarcado gitano.

\section{LAS TRAYECTORIAS QUE CRUZAN LA LÍNEA}

A menudo los relatos nos llevaron por caminos de culpabilidad y culpabilización cuando las mujeres se referían a sus aportaciones modernizantes al sistema de valores. Unas habían avanzado entre negociaciones, otras habían esperado su momento, algunas se habían alejado del grupo rompiendo con él, pero la mayoría continuaba avanzando hacia su objetivo de ser para sí, manteniéndose en la línea, "dando gusto" a unos y a otros sin perderse a sí mismas.

El concepto "trayectoria" permitió abordar estos relatos corales contenedores de vivencias temporales en los niveles intra-grupal, -en cuanto a rol, rituales de paso, compromisos asumidos, proceso enculturador, resistencia y crisis de identidad-, y exo-grupal -en cuanto a socialización, cambio, desajuste identitario y posición social.

Las trayectorias identificadas no son lineales; están cargadas de certezas y nuevos comienzos con un ayer de esfuerzo, atravesando la historia de su pueblo; donde la memoria colectiva relatada en primera persona es agente instituyente de identidad auto referenciada como grupo (Sanz, 1997). A la luz de los conceptos abismo generacional y ranuras de subjetividad (Bourdieu,1999) se pudieron interpretar las categorías que anidaban en las trayectorias, e inferir los rangos y posiciones de la mujer gitana en la estructura social patriarcal gitana, determinada por el sistema de valores y que dibujan dos grandes perfiles de trayectorias que se corresponden con sendas posiciones en el mapa identitario.

1.- Trayectorias de apayamiento necesario, donde las mujeres gitanas sin compromisos de rol han avanzado más lejos en la socialización; han establecido un vínculo intercultural más visible y durante la enculturación han mantenido cierto grado de reconocimiento intragrupal.

2.- Trayectorias de apayamiento estigmatizante y alienante, donde las mujeres gitanas con compromisos de rol han acusado interrupciones en su camino individual; han establecido vínculos interculturales más complejos 
y se perciben más alejadas del reconocimiento intragrupal.

Sin embargo, analizamos aquí tres tipos de trayectorias, las que se alejan, las que retornan y las que se mantienen en los alrededores de esa línea simbólica que ellas mismas trazan, partiendo de la descripción de la trayectoria tradicional/"modélica" de la gitanidad femenina.

\subsection{EL PUNTO DE REFERENCIA: LA TRAYECTORIA TRADICIO- NAL DE LA GITANIDAD FEMENINA.}

Como decíamos, el sistema de valores y sus mandatos es esencial para la pervivencia del sistema relacional gitano, sirviéndose de mecanismos de dominación replicados y congruentes con el patriarcado en el marco del paradigma tradicional; se retroalimenta de un sistema sexo-género (Amorós, 1985; 1996) con dinámicas androcéntricas de distribución de poder desigual y se refuerza dentro de la familia patriarcal, donde la mujer es artífice necesario como garantía de pervivencia de la raí que afectan a la individualidad en virtud de su posición social.

La adhesión voluntaria a esos valores y el cumplimiento de esos mandatos se percibe como inherente a la gitanidad; de modo que se espera que en el caso de las mujeres gitanas, la enculturación conduzca a su cumplimiento y preservación como parte de su misión universal. Es así que los marcos del sistema, posibilitan configurar trayectorias de referencia o modélicas de gitanidad en femenino representadas en la figura $1^{12}$.

${ }^{12}$ La figura 1 es una síntesis modelizada de la trayectoria de una mujer gitana joven observada durante cinco años. Parte de enculturación en entorno tradicional, con aprendizaje de rol asumido. En la escolarización ha socializado en el afuera, manteniéndose en el sistema educativo en tanto fue obligatorio (hasta los 16 años). El compromiso, la boda, el inicio de la vida conyugal en casa de los suegros, la observancia de la suegra, y la maternidad, han sido los hitos, el cumplimiento de los mandatos. La socialización educativa y laboral fueron prescindibles en tanto asumió su deber de rol en entorno tradicional. 


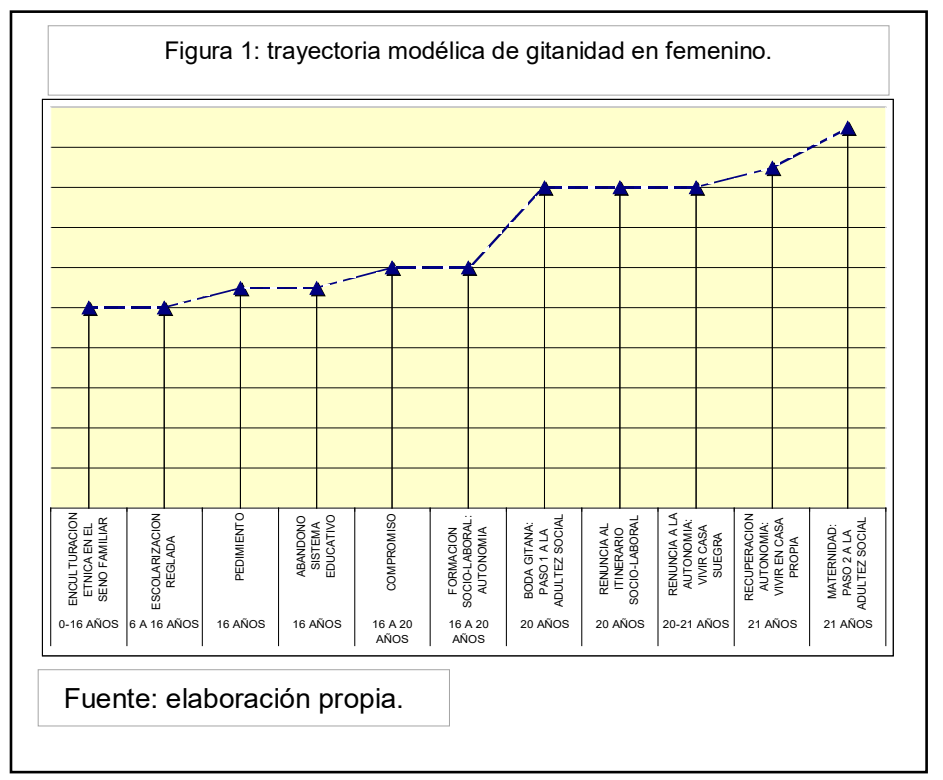

Un modelo análogo al de otras mujeres en entornos tradicionales ${ }^{13}$ de itinerario similar, bajo nivel de incertidumbre, merodeando el punto de cruce de línea: el de la cotidianeidad y la vida doméstica. Son trayectorias sin divergencias, ni abismos generacionales divergentes con los valores de género y los universales juveniles de rebeldía, autonomía (López, 1986) y auto-reflexividad en la modernidad (Giddens, 2000).

A partir de este modelo, se identificaron las trayectorias inesperadas de mujeres que no cumplen con lo que se espera de ellas y experiencias análogas al abismo generacional, convergentes en la tríada reflexión-decisión-acción, donde en fase autorreflexiva o autopoiética, han ido sufriendo crisis de adhesión o desidentificación en idénticas dosis. Son trayectorias impregnadas de rebeldía juvenil, más alejadas de la línea de cruce, con un sentido de identidad cultural propio y de identidad de género incipiente: dando espacio a la emergencia de una identidad proyecto (Castells, 2001) con voz femenina y gitana.

Las trayectorias divergentes perfilan una identidad de género como rito de paso en la evolución de la identidad cultural, cuestionando el sistema relacional, la distribución del poder intergéneros y el reconocimiento y legitimación del individuo en el intragrupo. La reflexividad emic de género ha buscado su propio camino, alejándose de limitaciones y afectaciones emocionales, llegando a romper un vínculo que han sentido estancado y del que han logrado salir viendo

13 En esta investigación para los Mandatos Obediencia y Respeto y respecto a la figura de la suegra, se tuvo en cuenta la perspectiva tradicional aplicada al estudio del entorno familiar de habitus musulman en Argelia a partir del análisis de Camile Lacoste-Dujardin en su libro Las Madres contra las Mujeres 
caer muchas vendas que no te hacen ver más allá (M.39), recuperando la identidad individual (étnico-cultural o de género) aun a riesgo de perder el sentido de pertenencia.

\subsubsection{Trayectorias que se alejan del umbral: divergencias extremas.}

Son trayectorias que se han detectado en voces de mujeres socializadas a ambos lados de la línea, que se han mantenido más tiempo en el sistema educativo y/o laboral. Revelaron haber sufrido un estigma alienante por apayamiento y culpabilidad por haber persistido en la construcción de su individualidad. Son mujeres que no han cumplido con lo que se esperaba de ellas ya sea porque no han tenido hijos como M.29 "no lo veía, la verdad, no quise que tuviéramos hijos”, contraviniendo los mandatos de respeto, sumisión y enculturación generacional. Ya sea porque no se han casado (anomalía sistémica provocadora de conflicto y dudas identitarias), ni lo harán hasta que la persona elegida las vea como "una mente pensante y también unas manos obrantes" afirmó M.30. Ya sea porque no han honrado al varón ("escapándose" con su novio gitano antes de la aprobación familiar, casándose con un payo o divorciándose) como en los casos de M.29, M.39 y R.01.

O finalmente, sea porque no han podido resistir la presión de los mandatos, alejándose hasta el punto de convertirse en islas autopoiéticas, pero conservando la referencia cultural en el origen. Su propuesta de solución a la tensión interna, ha sido "ir en solitario": una respuesta defensiva a la estigmatización alienante de apayamiento con firmeza en la identidad de género:

Acusarnos de apayadas es súper injusto. Es como que te excluyen. Te quieren decir que estás excluida totalmente. Es como decir: No perteneces. Te aceptamos pero no cuadras. $Y$, sí que a veces te puedes llegar a sentir mal. Pero, lo que pasa es que tienes que ser fuerte y un poco pesar en balanza qué es lo que más me compensa. En mi caso, lo que más me compensa a veces es estar en soledad (...) sé que están ahí, sé que si me pasara algo sé que yo puedo contar con ellos. Pero, ya me he acostumbrado durante estos diez años a sobrevivir yo sola a la adversidad, ¿no? por lo cual me he hecho súper independiente (...) (M.39)

En definitiva, son mujeres que se han enfrentado con la autoridad legitimante, desenmascarando el patriarcado y su estrategia de dominación bajo mandatos de respeto, sumisión y obediencia a la figura de autoridad legítima: el varón.

\subsubsection{Trayectorias de regreso: divergencias temporales.}

Se han detectado en las voces de mujeres autodefinidas "motor de cambio" que pretenden liderar un movimiento reflexivo que modifique y subvierta el reparto de poder en el sistema patriarcal gitano sin afectar los valores culturales autolegitimantes y estructurantes. Este discurso suele ser agradable en el exterior del grupo. La sociedad mayoritaria aprueba la modernidad gitana vestida de 
mujer. Y la mujer gitana que representa estos discursos ha encontrado apoyos externos y reconocimiento exogrupal antes que aprobación y reconocimiento intragrupal.

En la intersección entre exposición externa y deslegitimación interna el sujeto encuentra argumentos para validar su posición de referente, tirando del carro de su colectivo y apelando al sentido utilitario que enraíza en la cultura y posiciona el resultado de la tríada reflexión-decisión-acción como factor positivo que beneficiará al grupo. Al tiempo posicionan en el afuera un discurso reivindicativo y exigente por la missrecognition que redunda en una múltiple discriminación que carga más peso en la mochila personal durante el cruce de líneas.

Las mujeres que representan estos discursos están enculturadas en el grupo, socializadas a ambos lados y con estilos de vida interculturales (todas con trayectorias educativas y laborales de referencia, visibilizadas y ejerciendo un liderazgo étnico-cultural ajustado en la modernidad), posicionadas de manera autorreflexiva en el grupo, con una identidad cultural aggiornada que ha intentado integrar los estímulos internos y externos en los espacios de intercambio individual y grupal tanto en el adentro como en el afuera. Son mujeres expuestas -a veces extenuadas por sobreexposición- con fuerza para liderar la transformación de su colectivo pero con discursos que aún no alcanzan a los hombres. Estas mujeres muestran posiciones extremas: a) de ir con los hombres por la renovación del reparto de poder y del sistema sexo-género; b) de ir contra los hombres como única alternativa para subvertir el sistema relacional desigual, desmontar el androcentrismo y desenmascarar el patriarcado oculto detrás de mandatos autolegitimados como valores.

\subsubsection{Trayectorias que se mantienen en los alrededores: confluencia du- rante la fase de negociación.}

Estas trayectorias se han detectado en narradoras que esperan seguir siendo fieles a sí mismas y a su cultura, demandando tiempo en el adentro para el cumplimiento de mandatos y en el afuera para adaptar e integrar los estímulos modernizantes a su ritmo, en un tempo personal.

Van aquí tres ejemplos que abogan por esa confluencia entre el discurso autolegitimante de valores y los mandatos en fase de revisión, para dar espacio a la voz de género que se ha posicionado en el exogrupo como factor modernizador, análogo a la identidad proyecto, bisagra entre la identidad de resistencia y la legitimadora:

a) La posición de M.30 en los alrededores de la línea, sin arriesgarse a salir o alejarse demasiado del punto de salida: “(...) lo que tengo que trabajarme es una cultura que tiene unos valores muy bonitos, a los que no quisiera renunciar nunca". 
b) La posición de M.29 que ha construido su línea personal entre su propio adentro-fuera adhiriendo a la esencia de los valores.

Entender la gitanidad desde tu mirada, no es apayarse. Yo soy gitana digan lo que digan. Yo sé lo que soy, no voy a cambiar por la presión familiar (...) Soy fan del progreso, la igualdad de género y las personas que luchan por lo que quieren.

La de M.38 que elige salir, volver y merodear una recta que no dejará como sentido auto referencial:

(...) la cultura gitana es una recta, ¿vale? y yo sin desprenderme de la recta, he dado alguna curvita pero he vuelto a la recta (...) Haciendo cosas a los lados, pero sin salirme (...)

Estas tres posiciones reflejan un sentido de identificación étnico-cultural con el sistema de valores pero acusan cansancio por la persistencia de una estructura relacional desigual que camufla al patriarcado tras la defensa de los valores gitanos apoyados en mandatos o ritualidades. Estas voces han cargado responsabilidad al exogrupo, la sociedad mayoritaria legitimadora, por la tensión interna que sufren quienes deciden emprender un camino de construcción individual sin perder "la recta" de su cultura. Desde el afuera, se asume esa responsabilidad en cuanto al déficit de reconocimiento y la inclinación a sobre empatizar con las voces emergentes femeninas, exigiendo una aceleración del proceso de cambio, por lo que las narradoras han hablado de un necesario tiempo de adaptación para incorporar a estos individuos protagonistas de esa transformación como aliados que contribuyen a una mirada positiva, ya no a los individuos, que son ellas las mujeres, sino al colectivo.

\section{INCONCLUSIONES.}

En su lucha por alcanzar la individualidad en un ámbito de socialidad negociada, las mujeres de esta investigación han dado señales de hastío en algún caso, desidia en otros e incluso depresión por desistimiento. La cultura de la obligatoriedad les ha sometido a máxima tensión e incomprensión a ambos lados de la línea, cargando culpas, renuncias y simulaciones de aceptación de mandatos, "todo por no romper" o "por no salir de la manada". El proceso reflexióndecisión-acción no siempre ha sido comprendido por sus iguales, agudizando el dilema entre ser y deber ser. La estigmatización ha añadido angustia a la relación entre individuo y grupo.

Con esta investigación se ha intentado resolver conjuntamente con las mujeres, a través de su mirada, significados, algunas veces radicados en convicciones individuales, y otras, en convicciones de grupo. Puede captarse el conflicto interior entre: "sé lo que me pertenece" y "sé lo que quiero ser". Puede interpretarse ese conflicto en clave de la tensión del momento de cambio, en algún caso asumido en solitario y en otras sumando voluntades para el empeño.

De los relatos y desde la dimensión personal se han identificado discursos 
emergentes favorables a la modernización localizados entre las mujeres jóvenes que han participado de la investigación que se han mantenido más tiempo en la educación formal y han sido más permeables al contacto intercultural. Como portadoras de pesadas mochilas, han sido de observadas desde el endogrupo que midió o baremó su gitanidad, fueron acusadas de apayadas, sufriendo un simil de destierro ${ }^{14}$.

La identidad gitana está en un momento modernizado, modernizante y en constante actualización. Al mismo tiempo, la identidad de género en el patriarcado somete al sujeto mujer a la reflexión y priorización de los valores de una cultura viva que exige adhesiones y compromisos legitimados por la replicación y la continuidad histórica. Mientras no haya algo de mayor peso, la presión de la permanencia sigue radicada en el valor familia, con todas sus consecuencias.

La tensión del momento reflexión-decisión-acción empuja a cruzar la línea desde el interior de lo doméstico y la feminidad tradicional al contacto intercultural que demanda autonomía, auto-reflexión y en algún caso la autopoiesis del sujeto en la modernidad. La autorreflexión individual sobre la concepción de género hacia el intra grupo en comparación con lo que el género desde el exterior propone, hace que el cruce de líneas exija mayor determinación en el empeño.

En las trayectorias de paso a través del umbral se ha visto a quienes han llegado más lejos atravesando el estigma alienante, alienándose ellas mismas pero manteniendo un hilo conductor a la esencialidad de la raíz, que aún no representa un discurso de identidad proyecto y es excluido del control social en la resistencia. Pero la autorreflexión permite la reinterpretación de significados y valores, dando forma a una identidad exenta del grupo y no legitimada por ellos, pero personal y por necesidad, auto-legitimante.

En la dimensión personal, se han localizado dos focos de tensión durante el proceso reflexión-decisión-acción: reconocimiento y gestión del tiempo.

En el reconocimiento, donde se conjugan las miradas del adentro y del afuera, al alcanzarlo permite descansar en el tránsito por la línea y al cruzar el umbral mantener el grupo en el horizonte tranquiliza y minimiza el desasosiego. La aprobación de los suyos es lo que desvela a buena parte de estas mujeres gitanas que saben lo que les pertenece y que negocian y se esfuerzan por demostrarlo. Al mismo tiempo, el reconocimiento desde el exterior aleja al individuo al otro lado del umbral en el afuera y ralentiza la vuelta a casa -ámbito de la tradición-. En el afuera radica la percepción de una imagen distorsionada y exigente al sujeto en construcción, acelerando el proceso de auto reflexión y autopoiesis que se localiza en la intersección de la gitanidad, la femineidad, la modernidad y la tradición.

En cuanto a la gestión del tiempo, esta es clave en la emergencia del sujeto para la construcción de la identidad donde se produce una dicotomía existencial en la convivencia dentro de un sistema sexo-género divergente a un lado y otro de la línea, en la intersección entre paradigmas. En este foco hemos localizado la

${ }^{14}$ N. de i.: Destierro es una medida que refieren significar una sanción/dictamen de alejamiento temporal de un sujeto del grupo por causas de fuerza mayor y/o enfrentamiento familiar o entre familias.

EMPIRIA. Revista de Metodología de Ciencias Sociales. N. 38 septiembre-diciembre, 2017, pp. 41-62. ISSN: 1139-5737, DOI/empiria.38.2018.19705 
variable control social que mantiene al sujeto ligado a la estructura a través del sistema de valores; y de ahí emerge la conciencia emic de que alargar los plazos para cumplir mandatos, negociar su cumplimiento, decidir no actuar o incumplir con lo que se espera de ti, va dejando intersticios de subjetividad para la autoconstrucción individual dentro del grupo, apurando la modificación de habitus mientras se introduce la concepción de género durante el proceso. Es una tarea ardua y que requiere de mucha "mano izquierda", pero es donde más se testimonian avances, retrocesos, abandonos y renuncias.

La toma de decisiones y la gestión del tiempo frente a las alternativas individuales, son las caras de la voluntad y el empeño personal de superación, la elección añade dificultades como la minusvaloración de los iguales o "el apayamiento": "si hablas mejor: ala, apayada. Si te vistes mejor: mira la apayada. Si piensas más: apayada” se quejaba M.29.

Las mujeres que han participado en este estudio revelaron capacidad para desarrollar estrategias de negociación, una cualidad que explica que las decisiones no alejan de los compromisos culturales, y que los beneficios del desarrollo personal redundarán en la mejora de todos Mediante soluciones de compromiso van diseñando sus trayectorias y gestionando el tiempo según las prioridades. Reflexividad que construye una individualidad que asume el papel de referente capitalizándolo como herramienta de reconocimiento por parte del grupo: "Se me ha visto un poco como eso de referente y como una ayuda (...) He sabido ser un apoyo para los gitanos para que se sepan mover en la sociedad mayoritaria. Eso lo han visto, que yo he sabido mirar los papeles y he sabido rellenarlos y entonces para ellos eso es súper importante" (M.30).

El coste de avanzar les ha significado sacrificio personal y enjuiciamientos desde el intragrupo por riesgo de alejamiento o pérdida, que les ha permitido justificar interrupciones (necesarias) para no anteponer intereses individuales a los grupales: “(...) cuando me casé mi marido al principio veía bien que estudiara. Pero yo estudiaba en adultos y volvía a casa a las 10 de la noche y me dijo: jah, no! Y lo dejé, por amor lo dejé” (M.39).

La identidad proyecto emerge en las nuevas generaciones más arraigadas en el proceso de socialización y se posiciona como relato de una nueva gitanidad, donde se identifican narradoras auto definidas "motor de cambio" como agente transformador de las generaciones siguientes.

Será parte del desarrollo identitario y cultural el conjugar las voces disonantes que configuran una conciencia propia de género y que se encuentran posicionadas, primero, en los discursos de mujeres que apelan a la complicidad de los hombres y segundo, en las voces posicionadas contra los hombres, que dinamitan los resortes de la dominación patriarcal, embanderan la individualidad y la autonomía de decisión, abogando por un camino de respeto al sistema de valores independiente de mandatos.

En el resultado de esa convergencia situamos la posición que propone una solución a la encrucijada desde un sentido auto reflexivo, como trayecto apegado al sistema de valores, negociando los mandatos y retrasando su cumplimiento en tanto los intereses del grupo, la pervivencia cultural y la individualidad no entren 
en conflicto. Trayectos representados en el lema "quiero ser yo y seguir con mi cultura", demostrando capacidad para cruzar la línea, resolviendo la dialéctica individualidad-socialidad, aunque siempre navegando entre dos aguas.

\section{BIBLIOGRAFÍA}

ABAJO, J. y CARRASCO, S. (2004): Experiencias y trayectorias de éxito escolar de gitanas y gitanos en España, Madrid, Instituto de la Mujer.

AGUIRRE, J. (2006): Historia de las Itinerancias Gitanas, de la India a Andalucía, Zaragoza, Institución Fernando el Católico.

ALBERDI, I. (1996): "Nuevos Roles Femeninos y Cambio Familiar" en Sociología de las mujeres españolas, Madrid, Editorial Complutense, pp. 41-67.

AMORÓS, C. (Comp.) (1996): 10 Palabras claves sobre Mujer, Madrid, Akal Editores.

AMORÓS, C. (1985): Hacia una crítica de la razón patriarcal, Madrid, Anthropos.

BOURDIEU, P. (1999): La distinción: criterios y bases sociales del gusto, Madrid, Taurus.

BRETONES, E. (2008): Origen, género y generación. Jóvenes gitanas en las aulas: entre personas y culturas. Aproximación etnográfica a dicursos y prácticas educativas en el área de influencia de Barcelona, tesis doctoral, Universitat Autónoma de Barcelona.

BUSTELO, M. y LOMBARDO, E. (2005) "Mainstreaming de género y análisis de los diferentes marcos interpretativos de las políticas de igualdad en Europa: el proyecto MAGEEQ", Aequalitas, 17, pp. 15-26.

CASTELLS, M. (2000): La Era de la información, Madrid, Alianza, vol. 2

CHARMAZ, K. (2012). The Power and Potential of Grounded Theory. Medical Sociology Online, 6(3), 2-15.

DENZIN, N. y LINCOLN, Y. (2011): The sage handbook of qualitative research, California, Sage.

ELBOJ, C., y VICÉN, Mª J. (2007): "BRUDILA CALLÍ: las mujeres gitanas contra la exclusión. Superación del absentismo y fracaso escolar de las niñas y adolescentes gitanas", Flumen 10, pp. 45-74.

FLECHA, R., MIRCEA, A., SORDÉ, T. (2013): El pueblo gitano: una identidad global sin territorio, disponible en: http://ub.edu/geocritics [consulta: 16-12-2015]

FONSECA, I. (2009): Enterradme de pie. La odisea de los gitanos, Barcelona, Anagrama.

FOESSA (2014): VII Informe sobre exclusión y desarrollo social en España, disponible en http://www.foessa2014.es [consulta: 19-11-2015]

FRASER, N. (2001): "Repensar el reconocimiento: superar el desplazamiento y la reificación en las políticas culturales", en Cuestiones Actuales de Sociología del género, Madrid, CIS, pp. 29-42.

FSG (2011): "Población gitana, empleo e inclusión social. Principales resultados y conclusiones del Estudio comparado", en Cuadernos Técnicos 103, Madrid, FSG.

FSG, CEPG, MSPSS, LAPARRA, D. (2006): Hacia la Equidad en Salud: Estudio comparativo de las encuestas nacionales de salud a población gitana y población general de España, Madrid, MSPSS. 
GARCÍA-HUIDOBRO, R. (2016): La narrativa como método desencadenante y producción teórica en la investigación cualitativa, disponible en http://dx.doi.org/10.5944/ empiria.34.2016.16526 [consulta: 20-01-2017]

GIDDENS, A., (2000): Modernidad e identidad del yo. El yo y la sociedad en la época contemporánea, Barcelona, Península.

GIL, E. (1996): "Edad y Género (Aspectos demográficos, culturales e ideológicos)”, en Sociología de las mujeres españolas, Madrid, Editorial Complutense, pp. 17-40.

GOFFMAN, E. (1959; 2009): La presentación de la persona en la vida cotidiana, Buenos Aires, Amorrortu.

GLASER, B. (1992): Emergence vs Forcing: basics of Grounded Theory Analysis, California, Sociology Press.

HEREDIA, I. (2012): Control y exclusión social: la Ley de Vagos y Maleantes en el Primer Franquismo. Zaragoza, disponible en http://ifc.dpz.es/recursos/ publicaciones/28/93/08heredia.pdf [consulta 16-4-2015]

IBAÑEZ, J. (2007): "Cómo se realiza una investigación mediante grupos de discusión", en El análisis de la realidad social. Métodos y técnicas de investigación, Madrid, Alianza, pp. 283-297.

JIMÉNEZ, I. (2011): Cultura gitana en la España del siglo XXI, disponible en http:// www.revistalaocaloca.com/2011/01/cultura-gitana-en-la-espana-del-siglo-xxi/ [consulta 16-3-2015]

JIMÉNEZ, S. (2010): La mujer es el motor de cambio en la evolución de la sociedad gitana, disponible en http://www.gitanos.org/upload/64/66/fsg aragon.pdf [consulta 29-1-2017]

KAWULICH, B. (2005): La observación participante como método de recolección de datos, disponible en http://www.qualitative-research.net/fqs/ [consulta 29-1-2017]

LEBLON, B. (1985; 1993): Los gitanos de España. El precio y el valor de la diferencia, Madrid, Gedisa.

LOMBARDO, E. (2003): EU Gender Policy : Trapped in the "Wollstonecraft Dilemma"?, disponible en http://journals.sagepub.com/doi/ pdf/10.1177/1350506803010002003 [consulta 29-1-2017]

LÓPEZ, A. (1986): Los Bienatados, los jóvenes del casco viejo de Zaragoza, Zaragoza, IFC.

LUHMAN, N. (1997): Organización y decisión. Autopoiesis, acción y entendimiento comunicativo, Madrid, Anthropos.

MARTÍNEZ, A. (2007): La condición social y jurídica de los gitanos en la legislación histórica española (a partir de la Pragmática de los Reyes Católicos de 1499, tesis doctoral, Universidad de Granada.

MAIR, L. (1998): Introducción a la Antropología Social, Madrid, Alianza.

PATEMAN, C. (1987): The Patriarchal Welfare State: Women and Democracy, disponible en http://www.people.fas.harvard.edu/ ces/publications/docs/pdfs/CES WP7. pdf, [consulta 27-1-2017]

PLATERO, R. (2009): "Discriminación por orientación sexual e identidad de género", en Estudios interdisciplinares sobre igualdad, Madrid, Iustel, pp. 169-182.

PRIETO, O. (2007): La identidad gitana y su construcción panétnica: el caso gitano en Barcelona, tesis doctoral, Universitat de Barcelona.

PUJADAS, J. (2002): "El método biográfico, El uso de las historias de vida en ciencias sociales", en Cuadernos Metodológicos (5), Madrid, CIS. 
RAMÍREZ, J. (1971): Nosotros, los gitanos, Barcelona, Ediciones 29.

RODRIGUEZ, S. (2008): ROMIPEN, la identitat gitana. Aproximació filosòfica a la identitat de les persones de cultura gitana, tesis doctoral, Universitat Ramon Llul.

ROTHÉA, X. (2008): Construire la différence: élaboration et utilisation de l'image des gitans dans l'Espagne franquiste 1936-1975, tesis doctoral, Université Montpellier III.

SAN ROMÁN, T. (1997): La diferencia inquietante. Viejas y nuevas estrategias culturales de los gitanos, Madrid, Siglo Veintiuno.

SAN ROMÁN, T. (Comp.) (1994): Entre la marginación y el racismo. Reflexiones sobre la vida de los gitanos, Madrid, Alianza.

SAN ROMÁN, T. (1976): Vecinos Gitanos, Madrid, Akal.

SANZ, A. (2005): El método biográfico en investigación social: potencialidades y limitaciones de las fuentes orales y los documentos personales, disponible en doi: 10.3989/asclepio.2005.v57.i1.32 [consulta 27-1-2017]

SANZ, A. (1997): "Construyendo el silencio colectivo o la cara oculta de la memoria", Temas de Antropología Aragonesa 7, pp. 7-38

SAN MARTÍN, R. (2007): "La observación participante", en El análisis de la realidad social. Métodos y técnicas de investigación, Madrid, Alianza, pp. 144-165.

STRAUSS, A, (1997) Mirrors and Masks: The Search for Identity, New Jersey, Transaction Publishers.

STRAUSS, A, y CORBIN, J. (2002): Bases de la investigación cualitativa. Técnicas y procedimientos para desarrollar la teoría fundamentada, Medellín, Universidad de Antioquia.

TRINIDAD, A., CARRERO, V., SORIANO, R. (2005): Cuadernos Metodológicos 37, Madrid, CIS

VALLÉS, M. S. (2014): Cuadernos Metodológicos 32, Madrid, CIS

VALLÉS, M. S. (1997): Técnicas cualitativas de investigación social, Madrid, Síntesis. 